\title{
Resolution of bilateral ptosis after reduction of unilaterally elevated intraocular pressure in a child with Axenfeld-Reiger spectrum disorder.
}

\author{
Ketki Soin \\ Jefferson Medical College \\ Alex V. Levin \\ Wills Eye Institute, Thomas Jefferson University
}

Follow this and additional works at: https://jdc.jefferson.edu/willsfp

Part of the Ophthalmology Commons

Let us know how access to this document benefits you

\section{Recommended Citation}

Soin, Ketki and Levin, Alex V., "Resolution of bilateral ptosis after reduction of unilaterally elevated intraocular pressure in a child with Axenfeld-Reiger spectrum disorder." (2012). Wills Eye Hospital Papers. Paper 16.

https://jdc.jefferson.edu/willsfp/16

This Article is brought to you for free and open access by the Jefferson Digital Commons. The Jefferson Digital Commons is a service of Thomas Jefferson University's Center for Teaching and Learning (CTL). The Commons is a showcase for Jefferson books and journals, peer-reviewed scholarly publications, unique historical collections from the University archives, and teaching tools. The Jefferson Digital Commons allows researchers and interested readers anywhere in the world to learn about and keep up to date with Jefferson scholarship. This article has been accepted for inclusion in Wills Eye Hospital Papers by an authorized administrator of the Jefferson Digital Commons. For more information, please contact: JeffersonDigitalCommons@jefferson.edu. 


\title{
As submitted to:
}

\section{Journal of AAPOS}

\section{And later published as:}

\section{Resolution of bilateral ptosis after reduction of unilaterally elevated intraocular pressure in a child with Axenfeld-Reiger spectrum disorder}

\author{
Volume 16, Issue 3, June 2012, Pages 307-308
}

doi:10.1016/j.jaapos.2012.02.012

Ketki Soin, MD,a and Alex V. Levin, MD, MHSca,b

Author affiliations: aJefferson Medical College, Thomas Jefferson University, Philadelphia, Pennsylvania; bWills Eye Institute, Department of Pediatric Ophthalmology and Ocular Genetics, and Departments of Pediatrics and Ophthalmology, Thomas Jefferson University

We report a 9-month-old boy with bilateral pseudoptosis associated with elevated IOP. The patient had previously undergone bilateral trabeculectomies and Ahmed tube placement and right cataract extraction and penetrating keratoplasty. At presentation, the right eye IOP was $24 \mathrm{~mm}$ $\mathrm{Hg}$ and the left eye IOP was $32 \mathrm{~mm} \mathrm{Hg}$. Approximately 2 weeks after dorzolamide was added to the treatment regimen for the left eye, the mother reported that the ptosis had resolved. We suggest several explanations for the resolution of the ptosis.

\section{$\underline{\text { Case Report }}$}

A 9-month-old boy with a history of Axenfeld-Reiger spectrum and glaucoma presented to Wills Eye Institute for follow-up evaluation of glaucoma caused by anterior segment dysgenesis. His mother also reported that he had bilateral severe ptosis since birth and held his chin-up when trying to view straight ahead. The history was unclear as to whether this action was constant or intermittent or related to the course of the child's glaucoma. No previous health records were available. Glaucoma had been diagnosed in both the patient's eyes at birth. He had undergone two trabeculotomies in each eye with later Ahmed tube implantation in each eye as well as penetrating keratoplasty and cataract extraction in the right eye. At presentation, his glaucoma was being medically managed with topical timolol $0.5 \%$ twice daily in the left eye. Beyond his ocular problem, he had no significant medical history. There were multiple family members with Axenfeld-Reiger spectrum, including siblings and his father. 
On examination, the child was able to fixate and follow targets with either eye. He demonstrated a marked chin elevation and severe bilateral ptosis with only a minimal upper lid crease (Figure 1A). We could not reliably demonstrate any levator function. There was no lid swelling or erythema. He had large-amplitude, horizontal, symmetric, pendular, nystagmus, with no apparent null point. There was no strabismus, limitation of ocular motility, or photophobia.

At presentation, examination under anesthesia with handheld slit-lamp revealed a mild bleb over the right Ahmed plate and clear corneal graft with some sutures still in place, all of which were buried with no exposed knots and no overlying fluorescein staining. Corneal diameter was 12 $\mathrm{mm}$. The anterior chamber was deep and clear, with a $5 \mathrm{~mm}$ irregular pupil with posterior synechiae to an intact anterior hyaloid face. The left eye had a moderate bleb, paracentral corneal opacity consistent with a Peters anomaly variant, and corneal diameter of $12 \mathrm{~mm}$. The anterior chamber was deep with an oblong vertical pupil, extending limbus to limbus. IOP measured by Tono-Pen (Reichert Technologies, Depew, NY) was $24 \mathrm{~mm} \mathrm{Hg}$ in the right eye and $32 \mathrm{~mm} \mathrm{Hg}$ in the left eye. Central corneal thickness was $560 \mathrm{mmin}$ the right eye and $768 \mathrm{mmin}$ the left eye. No apparent corneal epithelial edema was found in either eye. The findings of a dilated fundus examination revealed a healthy posterior pole in both eyes with an estimated cup/disk ratio of approximately 0.6 in each eye.

To manage the elevated IOP in the left eye, timolol $0.5 \%$ eye drops were replaced with a combination of dorzolamide and timolol twice daily in the left eye. Two weeks later, the patient's ptosis and abnormal head posture had completely resolved (Figure 1B). Examination under anesthesia 1 month later revealed an IOP of $24 \mathrm{~mm} \mathrm{Hg}$ in the right eye and $22 \mathrm{~mm} \mathrm{Hg}$ in the left eye. Corneal pachymetry was not performed. In more than 20 months of subsequent follow-up, the ptosis has not recurred, and the IOP has been controlled, with pressures in both eyes $18-24 \mathrm{mmHg}$.

\section{$\underline{\text { Discussion }}$}

We present a case of severe ptosis that we believe resolved with medical management of glaucoma. The patient presented to us at 9 months of age. His mother reported that he had had ptosis since birth. The severity of the ptosis and the near absence of an upper lid crease suggested primary congenital ptosis. The rapid correction of the ptosis in our patient after 2 weeks of glaucoma treatment, however, ruled out that diagnosis.

Before we initiated care, the patient had undergone several surgeries in both eyes to control his glaucoma and cataract extraction in the right eye. It was unclear from the history whether the ptosis had ever resolved with these interventions. Transient or persistent postoperative ptosis can be a complication of eye surgery. The causes include photophobia, foreign body sensation, eyelid edema, hematoma, local anesthesia myotoxicity, and the use of a lid speculum. Ptosis has not been reported as a sign of pediatric glaucoma, although congenital/infantile glaucoma can present with the classic triad of photophobia, epiphora, and blepharospasm. The ptosis in our patient occurred in the absence of these signs. Pseudoptosis has been reported in adult patients with photophobia.1 
The only change made in our patient's treatment between presentation and the resolution of his ptosis was the addition of dorzolamide to the eye with elevated IOP, and the only observed effect other than the resolution of ptosis was the lowering of IOP in that eye. It is unlikely that the eye drops had a pharmacologic effect on the superior tarsal muscle or the levator palpebrae superioris, causing the lids to open more fully. Dorzolamide, a carbonic anhydrase inhibitor, does not have any sympathomimetic effect to stimulate the levator or M€uller's muscles.

It is possible that the ptosis, if a postoperative complication or transient phenomena of unknown other etiology, coincidentally resolved at the same time we were managing his glaucoma. The speed of resolution and the severity of the ptosis, however, argue against a surgical injury. The child had no manifestations of a systemic disorder, such as myasthenia gravis, that can cause transient ptosis. The fact that there was no later recurrence of the ptosis after its resolution supports the absence of an underlying systemic disease.

The rapid correction of the ptosis combined with the decrease in IOP after 2 weeks of new glaucoma treatment led us to believe that controlling the patient's glaucoma was responsible. Pseudoptosis has not been reported as a common symptom of congenital glaucoma. It is not specific to glaucoma, nor is it the sole presenting manifestation of congenital glaucoma. In a previously reported case, a patient with congenital glaucoma and pseudoptosis slept during the day, played in the dark, and hid his face during examinations because of extreme photophobia. 2 In that case, adequate control of the patient's glaucoma resolved the photophobia and the pseudoptosis.

Our case raises the possibility that severe ptosis may be a rare manifestation of uncontrolled glaucoma caused by anterior segment dysgenesis. Ophthalmologists should consider that possibility even when the ptosis is severe.

\section{$\underline{\text { References }}$}

1. Stone J. Pseudo-ptosis. Pract Neurol 2002;364-5.

2. Barkan O. Goniotomy for the relief of congenital glaucoma. Br J Ophthalmol 1948;32:701-28. 


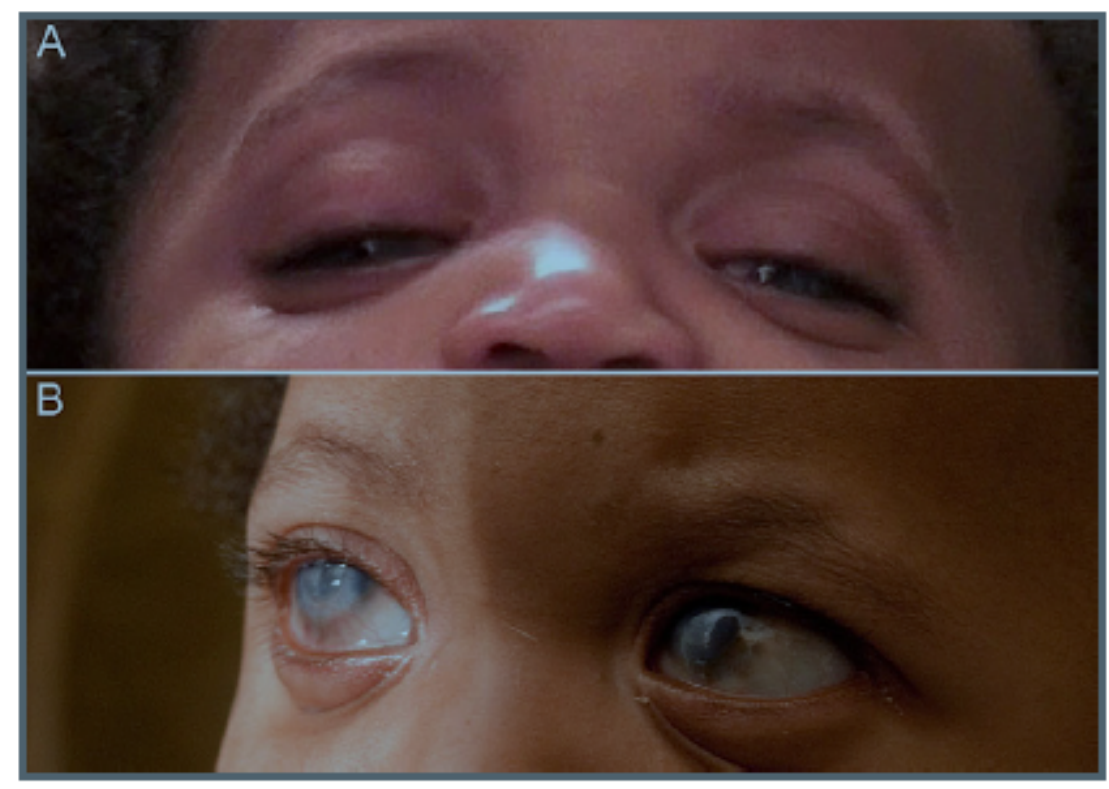

External photographs obtained before and after reduction of intraocular pressure. A, marked chin elevation and severe bilateral ptosis with only a minimal upper lid crease; B, 2 weeks later, the patient's ptosis and abnormal head posture completely resolved. 